\title{
ПРАКТИЧЕСКИЕ РЕКОМЕНДАЦИИ ПО ЛЕКАРСТВЕННОМУ ЛЕЧЕНИЮ ПЕРВИЧНЫХ ОПУХОЛЕЙ ЦЕНТРАЛЬНОЙ НЕРВНОЙ СИСТЕМЫ
}

Коллектив авторов: Кобяков Г.Л., Абсалямова О.В., Бекяшев А.Х., Голанов А.В., Измайлов Т.Р., Коновалов А.Н., Насхлеташвили Д.Р., Потапов А.А., Рыжова М.В., Смолин А.В., Трунин Ю.Ю., Улитин А. Ю., Усачев Д.Ю.

DOI: $10.18027 / 2224-5057-2020-10-3 s 2-07$

Ключевые слова: опухоли центральной нервной системы, коделеция 1p/19q, IDH-1,2 мутации, метилирование MGMT, первичная лимфома ЦНС, эпендимома, герминома ЦНС, медуллобластома, пинеобластома

\section{1. КЛАССИФИКАЦИЯ И ОПРЕДЕЛЕНИЕ СТАДИИ}

Гистологическая классификация первичных опухолей центральной нервной системы (ЦНС) проводится в соответствии с международной классификацией ВОЗ опухолей ЦНС от 2016 г. ТNМ классификация и стадирование в отношении первичных опухолей ЦНС не применяются. Международную гистологическая классификацию опухолей ЦНС (ВО3, 2016 г.) можно найти на сайте клинических рекомендаций Минздрава России: http://cr.rosminzdrav.ru/\#!/schema/831

\section{2. ДИАГНОСТИКА}

Диагноз первичной опухоли ЦНС основывается на данных анамнеза, осмотра, неврологического статуса, результатах инструментальных методов обследования (прежде всего, данных нейровизуализации - МРТ и КТ головного и / или спинного мозга) и патоморфологическом заключении.

\section{1. Жалобы и анамнез}

У большинства пациентов с опухолями ЦНС (опухолей головного и спинного мозга, далее сокращенно - ОГМ) заболевание проявляется в виде различных форм неврологических нарушений, что требует организации специальной этапности медицинской эвакуации, отличной от других опухолей. Неврологические нарушения могут быть острыми, в виде судорожного синдрома как проявления нарушения сознания на фоне гипертензионно-гидроцефального

Цитирование: Кобяков Г.Л., Абсалямова О.В., Бекяшев А.Х., Голанов А.В., Измайлов Т.Р., Коновалов А.Н. и соавт. Практические рекомендации по лекарственномулечению первичных опухолей центральной нервной системы. Злокачественные опухоли: Практические рекомендации RUSSCO \#3s2, 2020 (том 10).07 
синдрома, инсультоподобными, вследствие кровоизлияния в опухоль, или нарастать постепенно в форме нарастающей головной боли, проявления тех или иных неврологических выпадений (парезы и параличи, нарушения зрения, слуха, нарушения координации, статики и походки, и пр.). У части больных заболевание длительно протекает бессимптомно и обнаруживается зачастую спонтанно, при обследовании в связи с черепно-мозговой травмой или при профилактических обследованиях.

В случае острого начала заболевания пациенты должны быть госпитализированы в неврологическое или нейрохирургическое отделение стационара с возможностями инструментального обследования головного и спинного мозга (МРТ и/или КТ головного мозга). После выполнения КТ и/или МРТ при подозрении на опухолевое поражение головного (или спинного) мозга необходимо организовать консультацию врача-нейрохирурга с целью определения показаний и вида нейрохирургического вмешательства. Требования к выполнению МРТ головного мозга у нейроонкологических пациентов изложены ниже (см. 2.4.1).

\section{2. Неврологический осмотр}

Поводом для обращения к врачу у больных с опухолями мозга чаще всего является возникновение общемозговой или очаговой неврологической симптоматики.

К общемозговым синдромам и симптомам относят:

- различные формы нарушения сознания, как в виде снижения уровня сознания от оглушения до комы, так и в виде преходящих нарушений сознания (например, как единственное проявление судорожного синдрома);

- синдром внутричерепной гипертензии (развивается как следствие различных внутричерепных объемных процессов: очаг травматического ушиба головного мозга, опухоль головного мозга, кровоизлияние, водянка мозга (гидроцефалия) и др.); проявляется интенсивными головными болями, часто сопровождающимися приступами тошноты и рвоты, может также сопровождаться затруднением зрения вследствие отека дисков зрительных нервов на глазном дне;

- синдромы аксиального и тенториального вклинения головного мозга угрожающего жизни состояния, развивающегося как проявление синдрома внутричерепной гипертензии вследствие нарастающего отека головного мозга с ущемлением его в вырезке мозжечкового намета и / или в большом затылочном отверстии; проявляются в форме нарастающего снижения уровня сознания, ограничения взора вверх, в стороны;

- головные боли иной природы (например, вследствие раздражения оболочек, или гемодинамически значимых вторичных сосудистых нарушений);

- судорожный синдром - частое проявление поражения головного мозга, главным образом его супратенториальных структур, в части случаев - 
с признаками локализации патологии (локальных джексоновских припадков или характерной ауры перед приступом, чаще - при височной эпилепсии).

Очаговая неврологическая симптоматика разнообразна и представлена нарушением или выпадением различных функций в зависимости от локализации патологического процесса в головном мозге:

- судорожный (эпилептиформный) синдром в различных проявлениях;

- нарушение или ограничение движений в конечностях;

- снижение или выпадение чувствительности;

- нарушения высших корковых функций - речи, письма, счета, памяти;

- психопатологическая симптоматика - изменение поведения, неопрятность, продуктивная психопатологическая симптоматика и др.;

- нарушения зрения, слуха, обоняния;

- двоение перед глазами;

- асимметрия лица, нарушения фонации, глотания;

- болевые синдромы (например, тригеминальные боли на лице или таламические боли в конечностях).

Итак, из общемозговой и очаговой неврологической симптоматики складываются жалобы и предположительный диагноз опухолевого поражения головного мозга. Однако нельзя забывать и о важности данных анамнеза. Имеют значение давность появления тех или иных симптомов, очередность их возникновения, в части случаев - семейный анамнез болезней ЦНС и т. п. Так, кратковременная потеря сознания может быть вызвана эпилептиформным (судорожным) припадком, окклюзионным приступом или субарахноидальным кровоизлиянием (чаще сосудистого, а не опухолевого генеза). Особенности развития этого состояния могут сориентировать в предположительном диагнозе: эпилептиформные приступы чаще всего сопровождаются судорогами в конечностях половины тела или генерализованными судорогами, после выхода из этого состояния может отмечаться головная боль, но, как правило, умеренная, в отличие от сильной головной боли, дополняющейся картиной менингеального синдрома, при субарахноидальном кровоизлиянии.

При подготовке пациента к нейрохирургическому вмешательству необходима оценка его соматического статуса (консультация терапевта), а также общего функционального статуса (в нейроонкологии используется оценка функционального статуса по шкале Карновского).

\section{3. Лабораторная диагностика}

Рекомендуется выполнять развернутые клинический и биохимический анализы крови, определение РЭА, исследование свёртывающей системы крови, анализ мочи. Исследование свертывающей системы крови и общий анализ мочи обязательно выполняется перед хирургическим лечением, у остальных больных - при наличии клинических показаний. Конкретный набор параме- 
тров биохимического анализа крови и коагулограммы должен определять лечащий врач индивидуально.

При подозрении на герминативно-клеточную опухоль (например, при опухоли пинеальной области) выполняются анализы крови на опухолевые

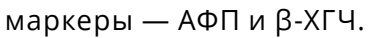

\section{4. Инструментальная диагностика}

Стандартом диагностического обследования пациентов с первичными опухолями ЦНС является выполнение МРТ головного мозга с контрастным усилением в указанных ниже режимах.

2.4.1. МРТ головного и /или спинного мозга:

- MPT головного мозга в трёх проекциях в стандартных режимах (T1, T2, FLAIR, T1 + контраст);

- функциональная MPТ двигательных и речевых зон; и МР-трактография; МР-ангиография, МР-спектроскопия, МР-перфузия;

- при подозрении на опухоль с риском диссеминации по оболочкам головного и спинного мозга (медуллобластома, пинеобластома, первичные герминативно-клеточные опухоли ЦНС и Ір.) выполняется МРТ всего спинного мозга с контрастным усилением;

- в случаях, когда МРТ не может быть выполнена (например, при наличии кардиостимуляторов или ферромагнитных имплантов), выполняется КТ с контрастным усилением и без него;

- КТ головного мозга выполняется также в дополнение к МРТ в случаях костных опухолей, при показаниях - КТ-перфузия, КТ-ангиография.

2.4.2. ПЭТ/КТ головного мозга с аминокислотами (метионином, тирозином, метилдопа) является возможной опцией (выполнение зависит от доступности данного вида исследования).

2.4.3. ЭЭГ обязательна у пациентов с судорожным синдромом.

2.4.4. Следующие инструментальные методы обследования также являются обязательными при подготовке пациента к хирургическому вмешательству:

- УЗИ вен нижних конечностей (для исключения тромбоза), УзИ внутренних органов (для оценки соматического статуса и ориентировочного скрининга других онкологических заболеваний);

- рентгенография и / или КТ органов грудной клетки (в том числе для исключения COVID19-ассоциированной пневмонии) 2;

- ЭКГ и ЭхоКГ - по показаниям (при наличии кардиоваскулярных заболеваний, возрасте старше 60 лет, предполагаемой операции в положении «сидя», при планировании лучевой и /или химиотерапии);

- консультация офтальмолога (оценка глазодвигательных, зрительных нарушений, а также признаков внутричерепной гипертензии на глазном дне); 
- консультация оториноларинголога (обязательна в случае планирования трансназального доступа).

\section{5. Морфологический диагноз}

Формулировка морфологического диагноза согласно МКБ-О должна включать в себя пятизначный код опухоли в соответствии с классификацией ВОХ опухолей ЦНС (2016 г.), четырехзначный код локализации опухоли, а также степень злокачественности (G). Для эпидемиологического учета опухолей следует указывать четырехзначный код согласно МКБ-10.

Дополнительно выполняются следующие молекулярно-генетические исследования.

Для глиом - мутации IDH 1,2, коделеция 1p/19q, метилирование гена MGMT. При отсутствии технической возможности определения каких-либо из данных мутаций используется аббревиатура «NOS» (или БДУ - «без дополнительного уточнения»).

В случае лимфомы или при подозрении на таковую - CD45, CD20, CD79a, MUM1, BCL-2, BCL-6, ИM Ki67.

В случае диагноза герминативно-клеточной опухоли дополнительно опре-

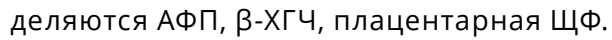

При выявлении эмбриональных опухолей (медуллобластомы и др.) выполняются дополнительные молекулярно-генетические исследования для субклассификации опухоли.

BRAF V600E-мутация выявляется в 3\% всех первичных опухолей ЦНС. Целесообразно ее исследовать при выявлении плеоморфной ксантоастроцитомы (и плеоморфной анапластической ксантоастроцитомы), а также пилоцитарной астроцитомы.

\section{3. СТРАТЕГИЯ КОМПЛЕКСНОГО ЛЕЧЕНИЯ ПЕРВИЧНЫХ ОПУХОЛЕЙ ЦНС}

Стандартными лечебными процедурами у больных с первичными опухолями ЦНС в настоящее время являются хирургическое лечение, радиотерапия, противоопухолевая лекарственная терапия. В целях обеспечения комплексного подхода к лечению пациентов со злокачественными глиомами необходимо формирование мультидисциплинарной «нейроонкологической команды» с участием нейрохирургов, радиотерапевтов, онкологов, а при необходимости - привлечение врачей смежных специальностей.

\section{1. Хирургическое лечение}

Хирургическое вмешательство в объеме удаления опухоли или стереотаксической (навигационной) или открытой биопсии образования является одним из основных методов комплексного лечения при ОГМ. Этот этап лечения выполняется в условиях специализированного (нейрохирургического) стационара. 
3.1.1. Удаление глиом головного и спинного мозга следует выполнять с применением операционного микроскопа и микрохирургической техники, в сопровождении интраоперационного нейрофизиологического мониторинга. Рекомендовано использование метаболической навигации с 5-аминолевулиновой кислотой, а также нейронавигационных устройств, включая УЗ, для интраоперационного уточнения расположения и распространенности опухоли. При необходимости применяются также эндоскопическая ассистенция, интраоперационная КТ или МРТ.

3.1.2. При планировании операции в положении «сидя» перед операцией необходимо проведение ЭхоКГ для исключения сообщения между предсердиями или между желудочками. В течение такой операции необходим контроль за показателями насыщения крови кислородом и углекислым газом с целью снижения риска клинически значимой воздушной эмболии.

3.1.3. При подозрении на герминому, первичную лимфому ЦНС удаление опухоли не показано. Рекомендовано выполнение стереотаксической (или навигационной) или открытой биопсии опухоли с последующим назначением комбинированного лечения.

3.1.4. Стереотаксическая или нейронавигационная биопсия выполняются с применением стереотаксических аппаратов или нейронавигационных устройств.

3.1.5. В послеоперационном периоде после удаления опухоли пациентам с опухолями ЦНС рекомендовано выполнение КТ головного мозга с контрастированием или без него и / или МРТ головного / спинного мозга с контрастным усилением или без него в течение 24-72 ч.

3.1.6. При рецидиве опухоли вопрос о показаниях к повторному удалению следует решать на консилиуме с участием нейрохирургов, радиотерапевтов, онкологов (химиотерапевтов) с учетом времени, прошедшего от предыдущей операции, анатомической возможности тотального удаления опухоли, наличия масс-эффекта, общего функционального статуса пациента, наличия и тяжести сопутствующих заболеваний.

\section{2. Лучевая терапия}

Лучевое лечение используется как самостоятельный метод терапии либо после хирургического удаления/биопсии опухоли, а также при возникновении рецидива/продолженного роста опухоли. лт может проводиться как на область опухоли (на остаточную опухоль или на ложе опухоли), так и на пути возможного метастазирования (например, краниоспинальное облучение (КСО) в лечении медуллобластомы), а также в качестве буст-терапии (дополнительное облучение опухоли /ложа опухоли). При лечении злокачественных опухолей (G III-IV) лт следует начинать в сроки от 2 до 6 нед. после операции, в ряде случаев - в комбинации с ХТ (см. раздел 3.3). 
Оптимальной методикой лт является применение конформных техник облучения, которое позволяет осуществить максимально безопасное подведение дозы на область опухоли с минимально возможной лучевой нагрузкой на здоровые ткани с учетом толерантности прилегающих структур. При планировании облучения пациентов с опухолями ЦНС проводят разметку с помощью мультиспиральной КТ, при возможности формируют индивидуальное фиксирующее устройство - термопластическую маску.

3.2.1. У пациентов с глиобластомой в возрасте до 65 лет режимом выбора послеоперационного лечения является режим ХЛТ с темозоломидом (рис. 4): ХЛТ с ежедневным приемом темозоломида (75 мг/м²) (в течение всего курса лТ - 30 фракций по 2 Гр на фракцию), с последующими 6-12 курсами темозоломида $5 / 23$ (см. раздел 3.3).

3.2.2. У пациентов с глиобластомой в возрасте старше 65 лет лт может проводиться в режиме 15 фракций по 3 Гр на фракцию, с одновременным приемом темозоломида.

3.2.3. Пациентам с анапластической астроцитомой рекомендованы либо лт с последующими курсами противоопухолевой лекарственной терапии в режиме PCV или в виде монотерапии (ломустин, темозоломид), либо режим ХЛТ с темозоломидом (см. раздел 3.3).

3.2.4. При наличии коделеции 1 p/19q (+/- мутация IDH-1,2) у пациентов с анапластической олигодендроглиомой лечение может быть начато как с лт, так и с XT (PCV или монотерапия темозоломидом² 5/23) Комментарий: в случае полного ответа на ХТ у пациентов с такими опухолями лТ может быть отложена в качестве резерва лечения при рецидиве (см. раздел 4.1.1.5, рис. 1).

3.2.5. При рецидивах глиом различной степени злокачественности тактика лечения определяется на мультидисциплинарном консилиуме (см. пункт 3.1.5). Виды лТ выбираются в зависимости от особенностей рецидива (локальный в месте первичной опухоли, отдаленный, диссеминация по оболочкам головного и/или спинного мозга и т. д.), времени от предыдущей лт, размера нового очага. Рекомендовано перед планированием повторной ЛТ выполнить ПЭТ /КТ с метионином/тирозином для уточнения распространенности опухоли.

3.2.6. Радиохирургическое лечение - одномоментное подведение высокой дозы (12 гр и более) с использованием стереотаксической техники. Используется у пациентов с небольшими (до 3 см в максимальном измерении) опухолями, имеющими чёткие границы (пилоидные астроцитомы, эпендимомы, менингиомы, невриномы, аденомы гипофиза, краниофарингиомы и пр.), а также при солитарных рецидивах злокачественных глиом (до 3 см в максимальном измерении).

3.2.7. Режим гипофракционирования (РОД $\geq 2,5$ Гр, СОД $\geq 15$ Гр) используется у пациентов с опухолями, имеющими чёткие границы, либо с минимальной зоной возможной инфильтрации тканей (до 3-5 мм), а также у пациентов 
с образованиями более 3 см и/или с опухолями, располагающимися вблизи критических структур мозга.

3.2.8. Краниоспинальное облучение (КСО) - обучение всего головного и спинного мозга - применятся у пациентов с опухолями, склонными к метастазированию по оболочкам головного и спинного мозга (медуллобластома, пинеобластома, негерминогенные герминативно-клеточные опухоли, анапластическая эпендиома с признаками диссеминации). Стандартным режимом дозирования при КСО является: РОД 1,6-2,0 Гр до СОД 36 Гр с бустом на ложе опухоли до СОД 54-55,8 Гр.

3.2.9. У пациентов с первичной лимфомой головного мозга, а также при диссеминации некоторых других опухолей используется режим облучения всего головного мозга с РОД 2 гр и СОД 40 гр.

3.2.10. У пациентов с первичной «чистой» герминомой головного мозга при стандартном комбинированном лечении после ХТ (см. ниже) выполняется облучение всей желудочковой системы РОД 1,8-2,0 Гр, СОД 24Гр. Дополнительно при наличии остаточной опухоли на буст до 40 Гр.

\section{3. Химиотерапия (противоопухолевая лекарственная терапия)}

Основные режимы ХТ первичных опухолей ЦНС представлены в табл. 1, 2, 3.

\section{Режимы лекарственной терапии первичных опухолей ЦНС}

Рекомендуемые режимы лекарственной терапии различных вариантов первичных опухолей ЦНС представлены в табл. 1, 2, 3.

Таблица 1. Рекомендуемые режимы лекарственной терапии глиом G II-IV

PCV: ломустин 90 мг/м² внутрь в 1-й день +винкристин 1,5 мг/м² в/в в 1-й и 8-й дни + прокарбазин
70 мг/м² внутрь в дни 8-21-й дни, каждые 6 нед.
Ломустин 110 мг/м² внутрь в 1-й день, каждые 6 нед.
Темозоломид 150-200 мг/м² в 1-5-й дни, каждые 4 нед. (5/23)
Темозоломид (в составе Хлт) 75 мг/м² ежедневно в течение лт (30 фракций по 2 Гр)
Темозоломид 150 мг/м² в 1-5-й дни + цисплатин 80 мг/м² (или карбоплатин АUС 3) в/в в 1-й день, каждые 4 нед.
Темозоломид 100 мг/м² в 1-8-й и 15-22-й дни, каждые 4 нед. (7/7)
Иринотекан 200 мг/м² в/в в 1-й и 15-й дни + бевацизумаб 5-10 мг/кг в/в в 1-й и 15-й дни, каждые 4 нед.
Ломустин 90 мг/м² внутрь в 1-й день + бевацизумаб 5-10 мг/кг в/в в 1-й, 15-й и 29-й дни, каждые 6 нед.
Ломустин 40 мг внутрь в 1-й, 8-й, 15-й и 22-й дни + бевацизумаб 5-10 мг/кг в/в в 1-й и 15-й дни
каждые 6 нед.
Бевацизумаб 5-10 мг/кг в/в в каждые 2 нед.


Таблица 2. Рекомендуемые режимы лекарственной терапии первичной лимфомы головного мозга

Метотрексат 3-8 мг/м² в/в в 1-й день (+ кальция фолинат 0,5 мг/кг в/в каждые 6 часов, начиная через 24 часа после введения метотрексата и под контролем снижения его концентрации в крови) + дексаметазон 8 мг/сут. в 1-3-й дни, каждые 3 нед.; проводится только в стационарных условиях онкогематологического отделения или отделения химиотерапии

Ритуксимаб 375 мг/м² в/в каждые 3 нед.

Темозоломид 150-200 мг/м² в 1-5-й дни каждые 4 нед. + ритуксимаб 375 мг/м² в/в каждые 3 нед.

Таблица 3. Рекомендуемые режимы лекарственной терапии первичных герминогенных опухолей ЦНС, эпендимомы, медуллобластомы, пинеобластомы

Цисплатин 25 мг/м² в/в в 1-4-й дни + этопозид 80 мг/м² в/в в 1-4-й дни, каждые 3 нед.

Цисплатин 20 мг/м² в/в в 1-5-й дни + этопозид 80 мг/м² в/в в 1-5-й дни + ифосфамид 1500 мг/м² (+ месна) в/в в 1-й, 3-й, 5-й дни, каждые 3-4 нед. (в зависимости от переносимости)

\section{4. АЛГОРИТМЫ ЛЕЧЕНИЯ РАЗЛИЧНЫХ ГРУПП ПЕРВИЧНЫХ ОПУХОЛЕЙ ЦНС}

В настоящем разделе представлены алгоритмы выбора тактики лечения в зависимости от нозологической группы: глиальные опухоли, медуллобластома, первичная лимфома ЦНС, первичные герминогенные опухоли ЦНС, паренхиматозные опухоли шишковидной железы (пинеобластома и пинеоцитома с промежуточной дифференцировкой), хориоидкарционома и др.

\section{1. Глиомы}

Традиционно подходы к лечению глиом в ведущих профессиональных сообществах в мире рассматривают в зависимости от степени злокачественности: глиомы низкой степени злокачественности (G I-II) и глиомы высокой степени злокачественности (G III-IV). На наш взгляд такой подход несколько устарел, особенно с накоплением знаний о роли IDH-1,2 мутаций в прогнозе и чувствительности к лучевой и противоопухолевой лекарственной терапии. Полностью пока не отказываемся от привычных схем, но сделаем некоторые важные акценты в алгоритмах выбора лечебной тактики.

\subsection{1. Глиомы низкой степени злокачественности (G I-II)}

В настоящее время установившееся деление глиом на группы «низкой степени злокачественности» G I-II и «высокой степени злокачественности» G III-IV представляется неактуальным в связи с выявлением IDH-1,2 мутаций. 
В данном разделе мы приведем алгоритмы лечения для глиом G II и некоторые особенности подходов к лечению более редких морфологических вариантов глиом.

\subsubsection{1. Ганглиоглиома, ганглиома, дизэмбриобластическая нейроэпителиальная опухоль (ДНЭО) (grade I)}

Перечисленные нозологические формы редких глиальных опухолей головного мозга в классическом варианте являются доброкачественными и относятся к опухолям G I. Основным их проявлением является судорожный синдром. Показано удаление опухоли (в части случаев с резекцией гиппокампа или иных эпилептогенных структур). Адъювантная терапия в случае радикального удаления опухоли не требуется. Следует отметить, что существует также понятие «анапластическая ганглиоглиома G III». Лечение этой опухоли осуществляется по принципам лечения злокачественных глиом. Дополнительно определяется BRAF мутация (в случаях выявления может быть назначена анти-BRAF терапия на основе вемурафениба или дабрафениба, которая эффективна в отношении этих редких опухолей).

\subsubsection{2. Субэпендимарная гигантоклеточная астроцитома (grade II)}

Чаще всего является одним из проявлений туберозного склероза (болезни Бурневилля). На МРТ представлена солидной опухолью, расположенной чаще всего в проекции одного или обоих отверстий Монро, гиподенсивной в Т1-режиме и интенсивно накапливающей контраст. На КТ характерно наличие симметричных кальцинатов в проекции зрительных бугров. Стандартом лечения этих опухолей является оптимальное (тотальное) удаление. Больным с диффузным поражением показано лечение эверолимусом.

\subsubsection{3. Плеоморфная ксантоастроцитома (G II)}

Плеоморфная ксантоастроцитома на МРТ - как правило, кистозная, хорошо отграниченная от окружающего мозгового вещества опухоль, со́лидная часть которой интенсивно накапливает контраст и расположена асимметрично относительно кисты. Показано удаление опухоли. Адъювантная терапия в случае радикального удаления опухоли не требуется. Следует указать, что в классификацию 2016 г. внесено понятие «анапластическая плеоморфная ксантоастроцитома G III». Лечение этой опухоли осуществляется по принципам лечения злокачественных глиом. Дополнительно определяется BRAF мутация (в случаях выявления может быть назначена анти-BRAF терапия на основе вемурафениба или дабрафениба, которая эффективна в отношении этих редких опухолей). 


\subsubsection{4. Пилоидная (пилоцитарная) астроцитома (ПА) (G I)}

Обычно встречается в детском возрасте и у молодых взрослых. Оптимальным методом лечения является хирургическое удаление (при условии операбельности опухоли). Обязательным является выполнение МРТ в послеоперационном периоде для оценки радикальности операции. В случае радикального удаления опухоли больному показано наблюдение. В случае остаточной опухоли показана ЛТ. Следует указать, что эта редкая группа опухолей неравномерна: выделяют ПА хиазмы и зрительных путей, пилоидную астроцитому срединных структур головного мозга (таламуса, среднего мозга и других отделов ствола мозга) и ПА мозжечка. Биологическое поведение ПА не всегда предсказуемо: некоторые из этих опухолей оказываются резистентными к лечению, иногда наблюдается диссеминация по оболочкам головного и/или спинного мозга.

\subsubsection{5. Астроцитома и олигодендроглиома (G II) больших полушарий головного мозга}

Предоперационный диагноз глиомы G II основан на MPT-признаках (гиподенсивная в T1 режиме, гиперденсивная в режимах T2 и FLAIR, не накапливает контраст) и клинических проявлениях (длительный анамнез, часто - судорожный синдром, медленное нарастание симптомов). Для олигоденроглиомы и олигоастроцитомы характерно наличие кальцинатов на КТ. Послеоперационное лечение (ЛТ, ХТ) зависит от радикальности удаления и факторов риска (рис. 1). Коделеция 1p/19q и IDH-1,2-мутации - прогностически благоприятные факторы.

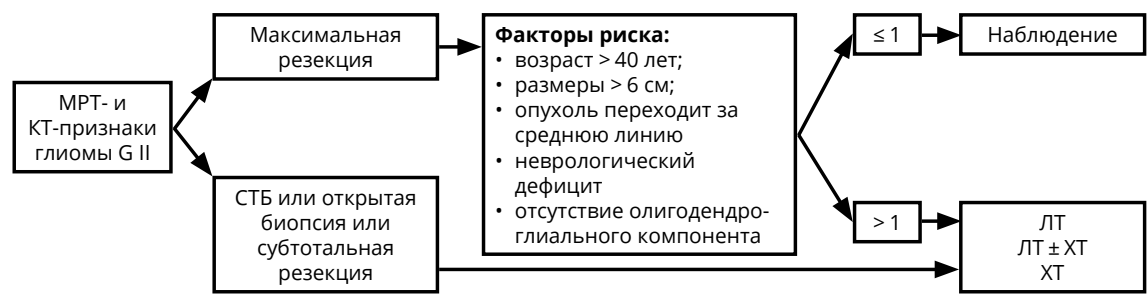

Рисунок 1. Рекомендуемый алгоритм лечения глиом G II.

В случае полной резекции опухоли и при наличии не более одного фактора риска показано наблюдение: МРТ 1 раз в 3-6 мес. или при нарастании симптомов (см. раздел 6 «Наблюдение»). При неполном удалении /биопсии опухоли или при наличии более одного фактора риска показано назначение лТ и/или ХТ.

Пациентам пожилого возраста (старше 70 лет) с обширными опухолями ЛТ может не проводиться (назначается монотерапия темозоломидом 5/23). Вопрос о назначении ЛТ и ХT ослабленным пациентам (с низким индексом 
Карновского и/или с признаками внутричерепной гипертензии), а также при большой распространённости опухоли решается индивидуально.

После окончания курса лТ должны быть назначены контрольные МРТ (через 2-4 нед. после окончания лт, а затем - каждые 2-3 месяца в течение 2-3 лет).

Рецидивы глиом G II требуют оценки возможности повторного хирургического лечения, а также изменения степени анаплазии (в случае повторной операции - по биопсии, или на основании данных ПЭТ /КТ с аминокислотами). При планировании лечения учитываются, какие методы были использованы ранее (применялись ли лучевая и/или химиотерапия, был ли достигнут полный ответ на лечение), а также их молекулярно-генетический профиль - наличие мутаций IDH 1,2, 1р/19q ко-делеции. При отсутствии последних, используются те же режимы лечения, что и при рецидивах злокачественных глиом.

\subsection{2. Низкодифференцированные глиомы (глиомы высокой степени злокачественности, G III-IV)}

После хирургического вмешательства и верификации диагноза астроцитарной глиомы grade III-IV показано продолжение лечения в виде лТ и XT (рис. 2). Молекулярно-генетическими факторами, определяющими прогноз, являются IDH-1,2 мутации, метилирование гена MGMT, коделеция 1p/19q (все - благоприятные). Для пациентов с глиобластомой режимом выбора послеоперационного лечения является ХЛТ с ежедневным приёмом темозоломида (75 мг/ ${ }^{2}$ ) (в течение всего курса Лт - 30 фракций по 2 Гр на фракцию), с последующими 6-10 курсами темозоломида 5/23 (см. раздел 4).

При первом контроле после комбинированной ХлТ глиомы (в течение 1 мес. после окончания ЛТ) можно наблюдать феномен псевдопрогрессии: увеличение контрастируемой части опухоли при отсутствии нарастания неврологических симптомов на фоне стабильных доз ГКС. В таком случае рекомендован МРТконтроль через 1-3 мес. (то есть через 2-3 мес. после завершения ЛТ) с целью окончательной оценки динамики болезни.

Для пациентов с анапластической астроцитомой, анапластической олигодендроглиомой возможны варианты лечения: ХЛТ с темозоломидом или лТ (30 фракций по 2 Гр на фракцию, без темозоломида) с последующими курсами XT (режим PCV или монотерапия ломустином или темозоломидом) (см. раздел 4). Последний подход рекомендован в случае выявления IDH-1,2 мутаций. 


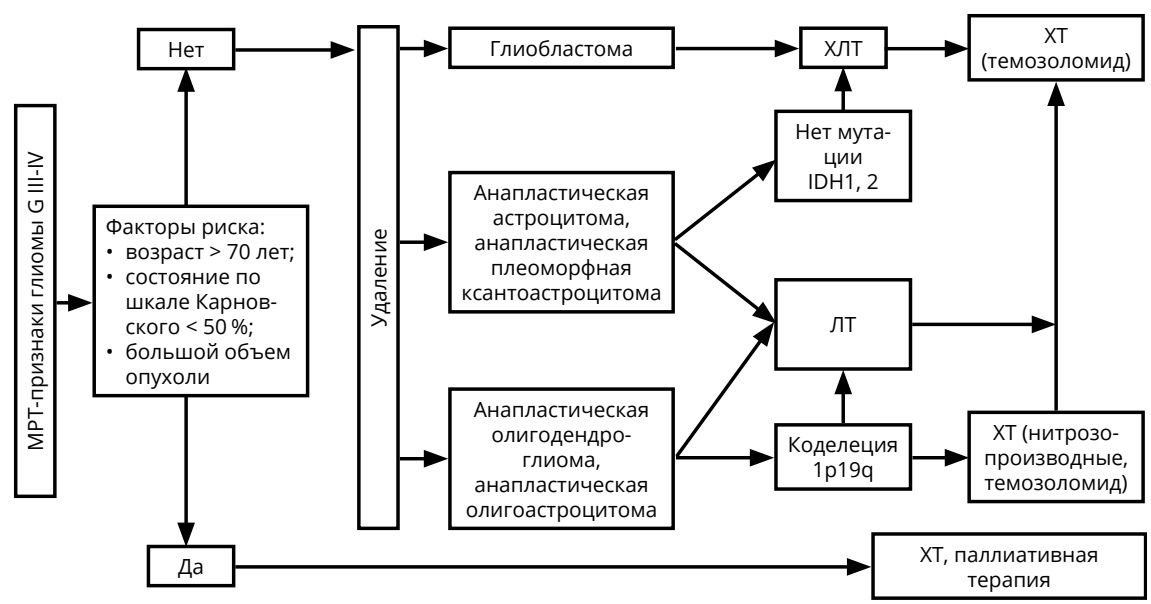

Рисунок 2. Алгоритм лечения злокачественных глиом G III-IV.

При выявлении коделеции 1р/19q у больных анапластической олигодендроглиомой в послеоперационном периоде могут быть назначены как лт, так и XТ (режим РCV или монотерапия темозоломидом 5/23). В случае полного ответа на химиотерапию лт может быть отложена до рецидива заболевания.

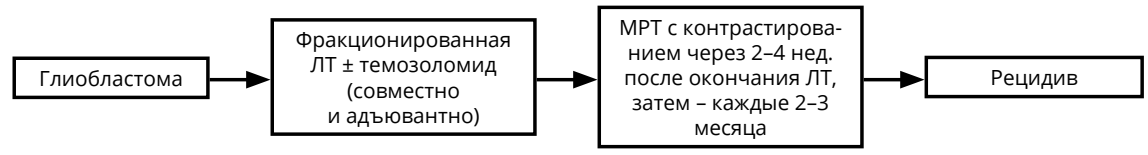

Рисунок 3. Схема химиолучевой терапии с темозоломидом.

\subsubsection{1. Рецидивы злокачественных глиом (G III-IV)}

Методами лечения рецидивов глиом являются повторное хирургическое вмешательство, системная XT, повторная ЛТ и паллиативная терапия. Показания к назначению любого из этих методов лечения должны обсуждаться мультидисциплинарно. В некоторых случаях может быть предложена повторная лт (если с момента предшествующей лт прошло не менее 8 мес.). При небольших локальных рецидивах могут рассматриваться радиохирургические методики или гипофракционированная лТ. 


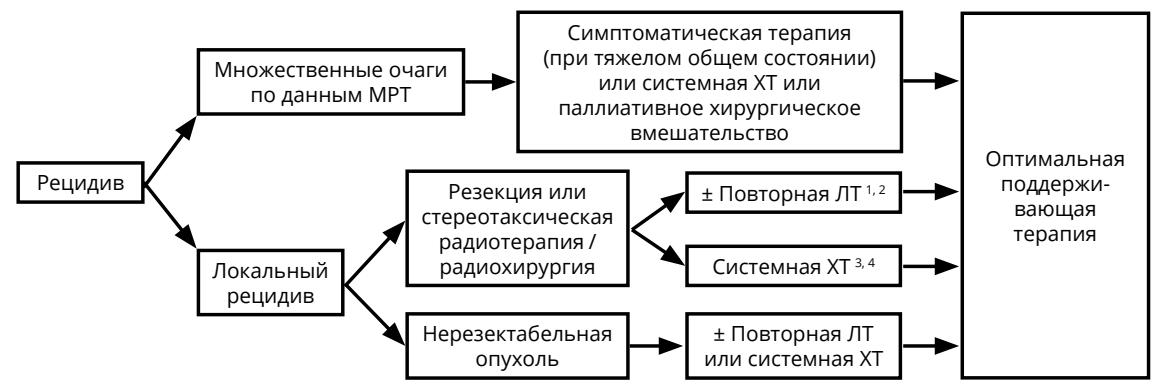

1 См. раздел 3.2.

2 Необходима МР-спектроскопия, МР-КТ перфузия, ПЭТ (с метионином) для исключения лучевого некроза, особенно, если интервал от первой Лт 18 мес.

3 При анапластической олигодендроглиоме и анапластической олигоастроцитоме предпочтительна ХT.

4 Ответ после двух последовательных неэффективных режимов ХТ маловероятен.

Рисунок 4. Рекомендуемый алгоритм лечения рецидивов злокачественных глиом.

В качестве ХТ рецидивов анапластических астроцитарных и олигодендроглиальных опухолей G III показано назначение темозоломида (если ранее не использовался или, если использовался, но был достаточно длительный - не менее 6 мес.- контроль болезни). Нитрозопроизводные могут также быть назначены, если они не использовались ранее. При олигодендроглиоме и анапластических олигоастроцитомах стратегия зависит от использованной ранее схемы лечения. В случае применения лТ назначается XT PCV; если использовались ЛТ и XT PCV, то предпочтение отдаётся ХТ темозоломидом. В случае первичной ХТ с использованием режима PCV, прежде всего, должна обсуждаться лТ. Если лТ не проводилась, то пациентам пожилого возраста, с обширным опухолевым поражением может быть предложена II линия ХT (например, темозоломид после PCV) (рис. 4).

\subsection{3. Глиомы ствола головного мозга}

При узловых формах опухолей ствола мозга, экзофитном характере роста, т. е. при МР-признаках полной или частичной отграниченности от структур ствола (например, при пилоидной астроцитоме) показано удаление опухоли (или открытая биопсия). Далее, в зависимости от гистологического диагноза, возраста и радикальности удаления назначаются ЛТ и/или ХТ. При диффузном характере роста опухолей ствола (например, при диффузной глиоме моста «диффузной понтинной глиоме») показано использование ЛТ и ХТ без обязательной верификации. При ювенильных глиомах четверохолмной пластинки после разрешения гидроцефалии осуществляется регулярное клиническое 
и МРТ-наблюдение (в случае прогрессирования опухоли рассматривается вопрос об удалении с последующей лт).

\section{2. Эпендимомы}

Редкие опухоли, возникающие из эпендимы головного мозга (чаще располагаются в пределах желудочковой системы, реже - экстравентрикулярно) или спинного мозга, примерно одинаково часто встречаются у детей и взрослых. Приблизительно в половине случаев на КТ и МРТ выявляются признаки мелких кальцинатов. Хорошо накапливают контрастное вещество, чаще - негомогенно. По классификации ВОЗ 2016 г. выделяют эпендимому (G II) и анапластическую эпендимому (G III).

Ведущую роль в лечении эпендимомы играет хирургическое удаление опухоли, поэтому при выполнении операции следует стремиться к максимальной резекции опухоли. При установлении гистологического диагноза эпендимомы или анапластической эпендимомы показано проведение МРТ головного и спинного мозга с контрастированием (с целью определения радикальности операции и выявления возможных субарахноидальных метастазов) и исследование спинномозговой жидкости на опухолевые клетки.

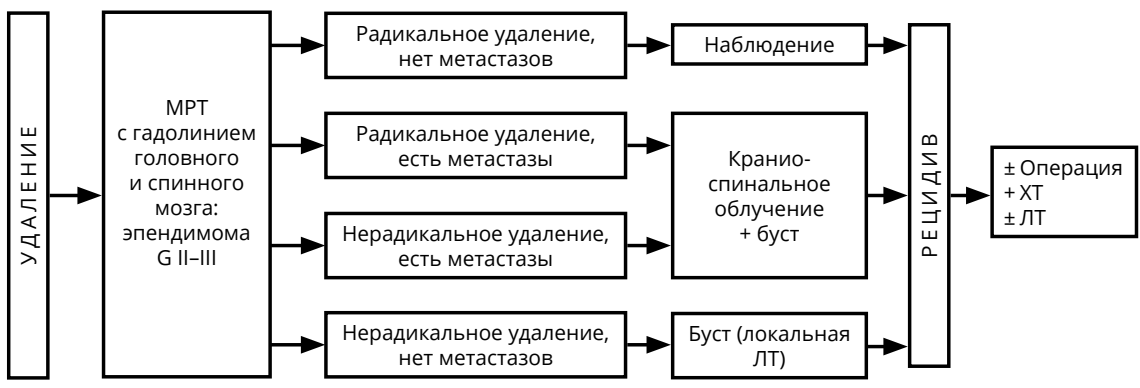

Рисунок 5. Рекомендуемая схема лечения эпендимом.

В случае выполнения максимальной резекции эпендимомы рекомендовано наблюдение (при отсутствии признаков метастазирования в виде наличия клеток опухоли в ликворе или спинальных метастазов на МРТ спинного мозга с контрастом). При невозможности радикальной операции или наличии резидуальной опухоли после хирургического вмешательства адъювантным методом лечения является ЛТ, объём которой определяется радикальностью операции и выявленной распространённостью опухоли. ХТ может использоваться в случаях рецидива эпендимомы (рис. 5). 


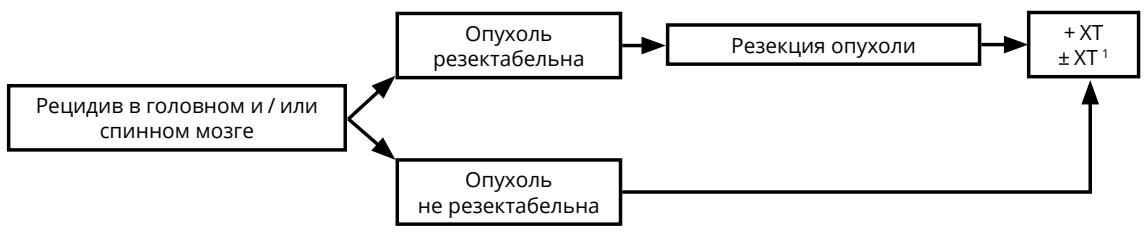

1 Объём лт зависит от объёма предшествующей лТ, распространённости опухоли и времени, прошедшего с момента предшествовавшей лт.

Рисунок 6. Рекомендуемый алгоритм лечения рецидива эпендимомы.

Контрольные обследования (МРТ с контрастированием и клиническое обследование) осуществляются не реже 1 раза в 3 мес. на протяжении первого года наблюдения, затем, при отсутствии данных за прогрессирование,- не реже 1 раза в 6 мес. В случае прогрессирования в виде солитарного очага рассматривается вопрос о повторной операции или, при небольших размерах рецидива, о радиохирургическом лечении (рис. 6).

\section{3. Первичная лимфома ЦНС}

Относительно редкая опухоль ЦНС (5-7\% всех первичных опухолей ЦНС), относящаяся, согласно онкогематологическим классификациям, к редким формам неходжкинских экстранодальных лимфом. Морфологически более чем в $90 \%$ случаев представлена диффузной В-клеточной лимфомой, но встречаются практически все описанные формы неходжкинских лимфом (Т-клеточная, внутрисосудистая крупноклеточная, MALT-лимфома твердой мозговой оболочки и др.).

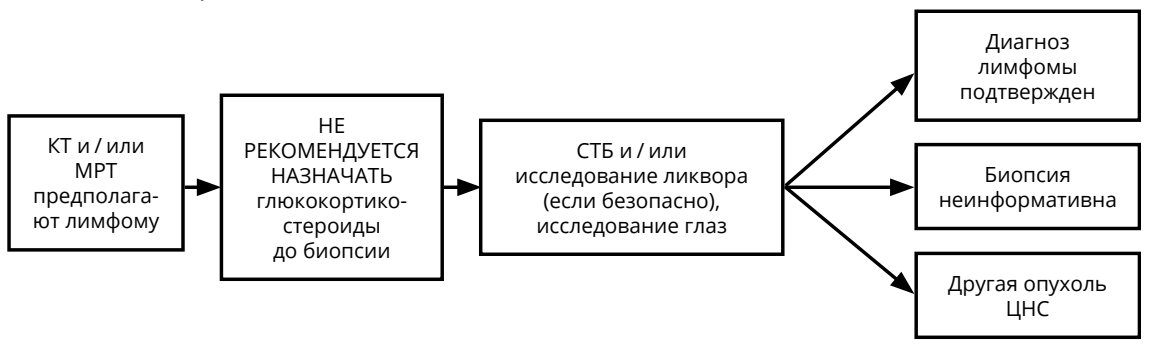

Рисунок 7. Рекомендуемый алгоритм диагностики первичной лимфомы ЦНС.

Различают лимфомы ЦНС у иммунокомпетентных пациентов и ВИЧ-ассоциированные лимфомы. У 15-20\% больных с первичными лимфомами ЦНС выявляется поражение опухолью стекловидного тела глаза. Методом выбора в установлении диагноза лимфомы ЦНС является СТБ. Прямая операция с целью 
верификации диагноза выполняется при локализации опухоли в опасных для выполнения СТБ областях мозга (например, мосто-мозжечковый угол, четвёртый желудочек, небольшая опухоль в проекции третьего желудочка и т. п.) или с целью разрешения угрожающих жизни состояний, обусловленных большим объёмом опухоли (рис. 7). После установления диагноза «первичная лимфома ЦНС» больной должен быть направлен в онкогематологический стационар для продолжения лечения. При общем состоянии по шкале Карновского > 50 \%, сохранной функции печени и почек, возрасте не старше 65 лет методом выбора

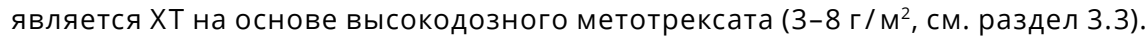
Данное лечение возможно только в условиях гематологического или химиотерапевтического стационара, располагающего возможностью контролировать уровень метотрексата в крови (рис. 8). Вариантом проведения высокодозной ХT с метотрексатом является внутриартериальная ХТ с временным открытием гематоэнцефалического барьера. У пациентов молодого возраста (до 50 лет) должен рассматриваться метод высокодозной ХT (на основе метотрексата) с пересадкой стволовых клеток крови. В случае полной ремиссии заболевания, сохраняющейся в течение года от момента верификации диагноза, пациент может быть оставлен под наблюдением. В случае неполного ответа показана ЛТ всего головного мозга в дозе 36 Гр (20 фракций по 1,8 Гр) с последующим проведением МРТ головы с в/в контрастированием и при необходимости - добавлением буста до СОД 45 Гр на сохраняющийся очаг накопления контрастного вещества. При лимфоме глаза показано дополнение лечения интравитриальным введением метотрексата и/или ЛТ поражённого глазного яблока (20 фракций по 1,8 Гр).

При рецидивах лимфомы ЦНС решение о методе лечения принимается исходя из нескольких параметров: времени с момента первичной верификации, продолжительности ремиссии, общего состояния больного, распространённости опухоли на момент рецидива (рис. 9). 


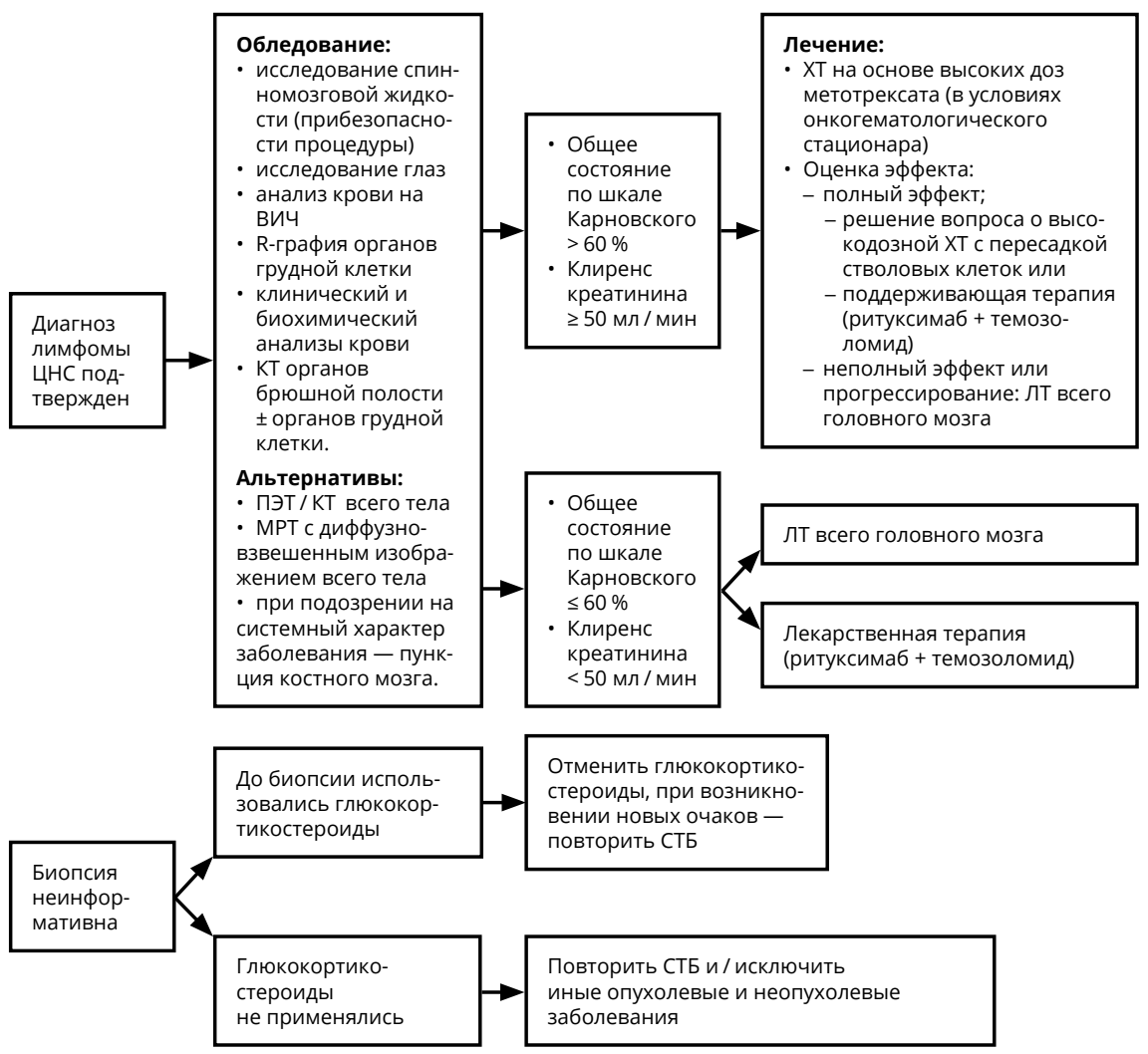

Рисунок 8. Рекомендуемый алгоритм лечения первичной лимфомы ЦНС.

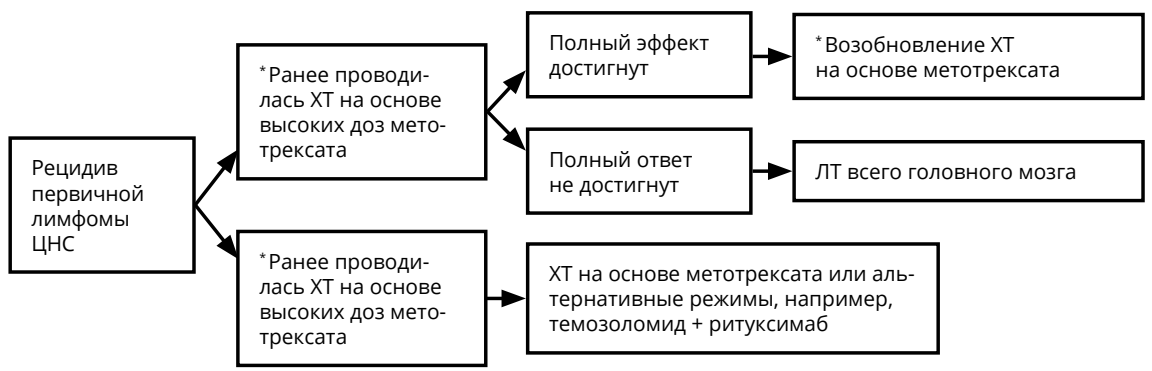

Рисунок 9. Рекомендуемый алгоритм лечения рецидива первичной лимфомы ЦНС. 


\section{4. Первичные герминогенные (герминативно-клеточные) опухоли ЦНС}

Редкая группа первичных опухолей ЦНС (0,9\% всех первичных опухолей ЦНС), встречается в основном в детском и молодом возрасте (медиана возраста на момент установления диагноза - примерно 17 лет), преобладает улиц мужского пола (в соотношении м/ж 7:1). Гистологически все разновидности этих опухолей идентичны аналогичным семиномным и несеминомным опухолям яичка и яичника.

В соответствии с Морфологической классификацией ВОЗ опухолей ЦНС 2016 г. выделяют следующие морфологические типы первичных герминативно-клеточных опухолей ЦНС:

- герминома;

- эмбриональная карцинома;

- опухоль желточного мешка;

- хориокарцинома;

- тератома:

- зрелая;

- незрелая;

- тератома со злокачественной трансформацией;

- смешанные герминативно-клеточные опухоли.

Самой частой локализацией первичных герминативно-клеточных опухолей ЦНС является пинеальная область, что обусловливает две типичные группы симптомов: окклюзионно-гидроцефальный синдром и глазодвигательные нарушения. По клиническому течению, формирующему подходы к лечению, разделяют герминому (используется также термин «чистая герминома» ЦНС) и негерминогенные герминативно-клеточные опухоли. Поскольку негерминогенные герминативно-клеточные опухоли ЦНС характеризуются повышением

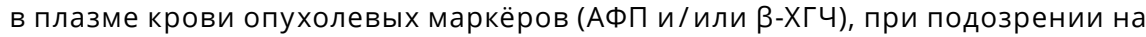
данные опухоли обязательно выполнение анализов крови на данные маркёры. Более того, при выявлении клинически значимого повышения одного или обоих маркёров (ХГЧ - более 5 нерм, АФП - более 2 норм) лечение начинают без гистологической верификации опухоли с ХТ. При нормальных уровнях маркёров показана верификация диагноза.

\subsection{1. Первичная ("чистая») герминома ЦНС}

Современный подход к лечению заключается в ХТ с использованием режима ЕР (цисплатин + этопозид) (см. раздел 3.3) с последующим облучением желудочковой системы головного мозга СОД 24 Гр (при отсутствии данных за метастазирование по оболочкам спинного мозга). Поражение хиазмальноселярной области требует выполнения анализов крови на гормоны гипофиза на всех этапах лечения с участием эндокринолога (лучше - нейроэндокринолога). Краткий алгоритм лечения больных с первичной герминомой ЦНС: 
- дооперационное обследование:

- МРТ головного и спинного мозга с контрастированием;

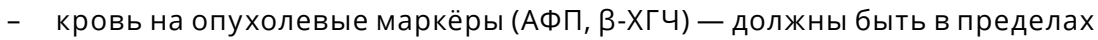
нормы;

- обследование у невролога, офтальмолога, эндокринолога;

- СТБ или прямая биопсия для верификации диагноза (при необходимости решение вопроса о ликворошунтирующей операции);

- $\quad$ ХТ по схеме ЕР (цисплатин + этопозид) - каждые 21 день;

- после 2 циклов - контрольная МРТ головного и спинного мозга с контрастированием;

- после 4 циклов - повторный контроль: МРТ головного и спинного мозга с контрастированием; при полном или «почти полном» эффекте - лт в объёме облучения всей желудочковой системы (до уровня С2 шейного позвонка): РОД 1,8-2,0 Гр, СОД 24 Гр; в случае подозрения на остаточную опухоль (вероятнее всего - зрелую тератому) до лТ решается вопрос об удалении;

- контрольная МРТ головного и спинного мозга каждые 3 мес. в течение первого года наблюдения, затем - каждые 6 мес. в течение 2-го и 3-го годов наблюдения, затем - 1 раз в год или при выявлении неврологическИх сИмптомов.

Эффективность такого лечения достигает $90 \%$ для 5-летней и 10-летней безрецидивной выживаемости, поэтому у фертильных пациентов мужского пола до начала лечения целесообразно решение вопроса о криоконсервации спермы.

\subsection{2. Первичные негерминогенные герминативно-клеточные опухоли ЦНС}

Это ещё более редкая, чем первичная герминома ЦНС, группа опухолей, неоднородная по спектру морфологических форм (эмбриональная карцинома, опухоль желточного мешка, хориокарцинома, тератома (незрелая, тератома со злокачественной трансформацией)), с менее благоприятным по сравнению с «чистой» герминомой прогнозом.

Краткий алгоритм лечения больных с первичными негерминогенными герминативно-клеточными опухолями ЦНС:

- дооперационное обследование:

- МРТ головного и спинного мозга с контрастированием;

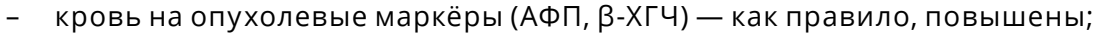

- обследование у невролога, офтальмолога, эндокринолога;

- ХТ по схеме «цисплатин + ифосфамид + этопозид» каждые 21 день;

- после 2 циклов - контрольная МРТ головного и спинного мозга с контрастированием, контроль опухолевых маркёров;

- после 4 циклов - повторный контроль: МРТ головного и спинного мозга с контрастированием, контроль опухолевых маркёров; при полном 
или почти полном ответе-лТ в объёме краниоспинального облучения: РОД 1,8-2,0 Гр, СОД 36 Гр; в случае подозрения на остаточную опухоль (вероятнее всего - одну из разновидностей тератомы) - до лт необходимо обсудить вопрос об удалении;

- контрольная МРТ головного и спинного мозга каждые 3 мес. (+ контроль опухолевых маркёров) в течение первых 2 лет наблюдения, затем - каждые 6 мес. в течение 3-го и 4-го годов наблюдения, затем - 1 раз в год или при выявлении неврологических симптомов.

5-летняя безрецидивная выживаемость при таком режиме лечения составляет 60-70\%.

\section{5. Медуллобластома}

Это злокачественная опухоль мозжечка, чаще встречающаяся у детей (самая частая злокачественная опухоль ЦНС у детей), и редко - у взрослых (обычно в молодом возрасте, но описаны случаи медуллобластомы у лиц старше 60 лет). С учетом локализации опухоли в различных структурах мозжечка, нередко с вовлечением IV-го желудочка и инфильтрацией ствола головного мозга, типичными проявлениями болезни являются гипертензионно-гидроцефальный синдром, нарушения вестибулярных функций и координации. Во всех ситуациях показано удаление опухоли. Радикальное удаление опухоли сопряжено с улучшением прогноза заболевания. Дальнейшее лечение медуллобластомы зависит от возраста пациента (дети младше 3-х лет, дети старше 3-х лет, взрослые - старше 16 лет), распространенности опухоли, признаков диссеминации по оболочкам головного и / или спинного мозга, особенностей молекулярно-генетического профиля. Мы рассмотрим стратегию лечения взрослых пациентов с медуллобластомой.

При подозрении на медуллобластому по клинико-рентгенологическим признакам больному показано выполнение МРТ всего головного и спинного мозга с контрастом еще на дооперационном этапе (рис. 9). Если до операции МРТ спинного мозга не выполнялось, то его обязательно следует выполнить в послеоперационном периоде при подготовке к лт, также выполняется анализ ликвора на опухолевые клетки. ЛТ проводится в режиме КСО с РОД 1,8-2,0 Гр и СОД 35 Гр с дополнительны бустом на ложе опухоли до 55 Гр. Во время ЛТ необходим контроль общего анализа крови из-за риска гематологической токсичности. С целью восстановления костного мозга по окончании лТ нужен перерыв в лечении в 4-6 недель до начала ХТ. В этот перерыв выполняется контрольная МРТ головного (и по показаниям - спинного мозга при выявлении очагов в спинном мозге перед ЛТ). 


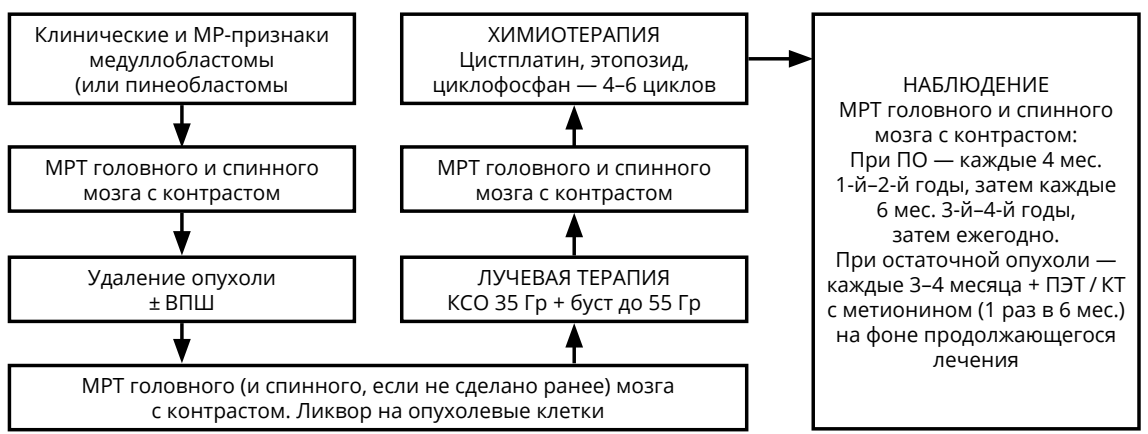

ВПШ - вентрикулоперитонеальный шунт, КСО - краниоспинальное облучение, ПО - полный ответ

Рисунок 9. Рекомендуемый алгоритм обследования и лечения медуллобластомы и пинеобластомы у взрослых.

ХТ проводится по схеме «цисплатин + этопозид + циклофосфамид» (см. табл. 3 раздела 3), каждые 4-6 недель (в зависимости от гематологической токсичности), всего 4-6 циклов с контрольными МРТ головного и спинного мозга после 3-х и 6-ти курсов лечения. В случае полного контроля болезни, рекомендовано наблюдение с контрольной МРТ головного мозга с контрастом каждые 3 месяца (+спинного мозга каждые 6 месяцев) в течение первых 2 лет, затем каждые 6 месяцев 3-й и 4-й годы, затем ежегодно. В случае остаточной опухоли выполняется ПЭТ/КТ с метионином (или с тирозином) и продолжается лечение с изменением схемы ХТ или с использованием ЛТ в режимах радиохирургии или гипофракционирования.

\section{6. Злокачественные опухоли пинеальной железы}

Опухоли пинеальной железы (ОПЖ) - редкие опухоли, возникающие из паренхимы шишковидного тела. Согласно классификации ВОЗ 2016 г., выделяются следующие ОПЖ:

- пинеоцитома (G I)

- аренхиматозная опухоль шишковидной железы промежуточной дифференцировки (G II-III)

- пинеобластома (G IV)

- папиллярная опухоль пинеальной области (G II-III).

Основными клиническими проявлениями ОПЖ является окклюзионно-гидроцефальный синдром и глазодвигательные нарушения. Пинеоцитома - доброкачественная опухоль, требует только хирургического удаления. Пинеоцитома с промежуточной дифференцировкой и пинеобластома - злокачественные опухоли, склонные к диссеминации по субарахноидальным пространствам 
и поэтому, как и при медуллобластоме, требующие выполнения контрастной МРТ всего головного и всего спинного мозга.

Тактика лечения схожа с таковой для медуллобластомы (см. раздел 4.5). У пациентов с пинеоцитомой с промежуточной дифференцировкой допустимо не проводить облучения всего длинника спинного мозга при отсутствии признаков диссеминации опухоли по субарахноидальным пространствам. У ослабленных пациентов со злокачественными ОПж лечение может быть начато с 2-4 курсов ХТ с последующей контрольной МРТ головного и спинного мозга и планированием лт.

\section{5. СОПРОВОДИТЕЛЬНАЯ ТЕРАПИЯ}

С первых проявлений заболевания и на протяжении практически всего курса лечения пациенты с опухолями ЦНС нуждаются в той или иной сопроводительной терапии, в зависимости от симптомов проявления болезни синдром внутричерепной гипертензии, наличие признаков угрозы вклинения головного мозга, судорожный синдром, цефалгия, эндокринные и электролитные нарушения и т. д.

\section{1. Кортикостероидная и дегидратационная терапия}

Основными противоотечными препаратами у больных с опухолями ЦНС являются ГКС (дексаметазон, преднизолон, солюмедрол и др.). Они показаны при нарастающем неврологическом дефиците и признаках отёка мозга (головная боль, спутанность сознания). ГКС могут быть причиной декомпенсации сахарного диабета, поэтому применять ГКС у больных сахарным диабетом следует с осторожностью и строго под контролем глюкозы крови, при необходимости - с коррекцией терапии. При подозрении на лимфому использование ГКС до гистологической верификации не рекомендуется за исключением ситуаций, когда отёк и дислокация головного мозга угрожают жизни больного. Интенсивность терапии зависит от выраженности симптомов; подбирается минимальная необходимая доза. После регресса симптомов необходимо постепенное снижение дозы вплоть до полной отмены (при отсутствии возобновления симптомов). Одновременно необходимо использовать гастропротекторы (Н2-гистаминные блокаторы или блокаторы протонной помпы). Салуретики (фуросемид), осмотические диуретики (маннитол) используются при выраженном отёке и дислокации мозга в комплексе с ГКС в условиях отделений интенсивной терапии под контролем электролитного состава крови.

\section{2. Противосудорожная терапия}

Антиконвульсанты назначаются при наличии судорожного синдрома (в т. ч. в анамнезе) или признаков эпилептиформной активности по данным ЭЭГ. При планировании и проведении ХТ желательно использование антиконвульсантов, 
не индуцирующих ферменты печени (ламотриджин, леветирацетам); нежелательно использование карбамазепина, фенитоина, фенобарбитала, а также вальпроата натрия (из-за его возможной гематологической токсичности). Профилактическое применение антиковульсантов в отсутствие эпиприпадков в анамнезе необязательно.

\section{3. Обезболивающая терапия}

Головная боль при опухолях головного мозга купируется преимущественно ГКС терапией, но важно, чтобы при подозрении на первичную лимфому ЦНС ГКС не назначались до верификации диагноза. При нейропатических болях требуется консультация невролога и/или психиатра. В остальных случаях используются НПВС, трамадол. Рекомендуется отмена НПВС за 5-7 суток до операции для снижения риска периоперационного кровотечения и контроль гемостаза с использованием тромбоэластограммы. При выраженном болевом синдроме, обусловленном поражением спинного мозга и/или позвоночника, показано применение наркотических анальгетиков (промедол, фентанил и др.).

\section{4. Коррекция гемостаза}

У пациентов с опухолями ЦНС наблюдаются различные отклонения в показателях гемостаза с преобладанием склонности к гиперкоагуляции. В периоперационном периоде для предупреждения ТЭЛА, начиная с третьих суток после операции, назначаются низкомолекулярные гепарины (надропарин кальция, эноксапарин натрия, далтепарин натрия и др.). При постоянном использовании пациентами антикоагулянтов/антиагрегантов (варфарин, клопидогрел, ацетилсалициловая кислота, фениндион, аценокумарол и т. п.) показан перевод на низкомолекулярные гепарины (под контролем тромбоэластограммы) не менее чем за 7 суток до операции с последующей отменой гепаринов за 12-24 часа до операции и возобновлением их введения через 1-2 суток. При выявлении в процессе лекарственной терапии тромбозов вен нижних конечностей определяют необходимость установки кава-фильтра, назначается терапия прямыми антикоагулянтами, противоопухолевая лекарственная терапия прекращается. По завершении приёма прямых антикоагулянтов лекарственную терапию опухоли можно возобновить, назначаются непрямые антикоагулянты на период 3-6 мес. с соблюдением осторожности при развитии тромбоцитопении.

\section{5. Коррекция гомеостаза}

У больных с поражением хиазмально-селярной области (глиомы хиазмы, герминогенные опухоли и пр.), следует контролировать водно-электролитные нарушения. При проявлении несахарного диабета - назначается антидиуретический гормон, при других проявлениях гипопитуитаризма - соответ- 
ствующая заместительная терапия. В связи с этим при лечении опухолей, располагающихся в хиазмально-селярной области, необходимо участие эндокринолога, желательно с опытом работы в области клинической нейроэндокринологии.

\section{6. НАБЛЮДЕНИЕ}

При достижении ремиссии (с полным ответом на лечение или при наличии остаточной опухоли) показано регулярное выполнение MPТ головного мозга (в трёх проекциях в стандартных режимах - T1, T2, FLAIR, T1 + контраст); для отдельных патологий - также MPT всех отделов спинного мозга с контрастированием. Оценка МР-данных должна производиться в соответствии с критериями эффективности лечения RANO. При глиомах G II MPT выполняется каждые 6 мес., при глиомах G III-IV - каждые 3-4 мес.; при первичной лимфоме ЦНС - каждые 3-4 мес. При первичных герминативно-клеточных опухолях, эпендимоме, медуллобластоме, пинеобластоме МРТ головного мозга с контрастированием и МРТ всех отделов спинного мозга с контрастированием выполняется каждые 3-4 мес. в течение первых 2 лет наблюдения, затем - каждые 6 мес. в течение 3-го и 4-го годов наблюдения, затем - 1 раз в год или при выявлении неврологических симптомов. У больных с первичными негерминогенными герминативно-клеточными опухолями регулярно проводится контроль опухолевых маркёров (АФП и ХГЧ). 